EPJ Web of Conferences 73, 07001 (2014)

DOI: $10.1051 /$ epjconf/20147307001

(C) Owned by the authors, published by EDP Sciences, 2014

\title{
Prospects for $\mathrm{K}^{+} \rightarrow \pi^{+} v \bar{v}$ observation at CERN
}

\author{
Stefano Venditti ${ }^{\mathrm{a}, \mathrm{b}}$ \\ CERN - 1211 Geneva 23, Switzerland
}

\begin{abstract}
The NA62 experiment at CERN aims at measuring the $\mathrm{K}^{+} \rightarrow \pi^{+} \nu \bar{v}$ decay Branching Ratio (BR) with unprecedented precision, by collecting $\mathrm{O}(100)$ events with $\sim 10 \%$ background in 2 years' data-taking. In this paper the measurement strategy adopted by the NA62 collaboration is described, as well as all the main detectors. The trigger system, a crucial item for the experiment, will also be discussed.
\end{abstract}

\section{Introduction}

The NA62 experiment [1] is the last from a long tradition of fixed-target Kaon experiments in the North Area (NA) of CERN. After a first phase dedicated to the measurement of the $\mathrm{R}_{K}=B R(K \rightarrow$ $e v) / \mathrm{BR}(K \rightarrow \mu \nu)$ ratio in 2007/8 [2] using the NA48 [3] experimental setup, the latter was dismantled (except for the liquid Krypton calorimeter) to allow the mounting and testing of new detectors, designed for the measurement of the $\mathrm{K}^{+} \rightarrow \pi^{+} \nu v$ decay BR. The experiment will start taking data in Fall 2014.

\section{The $\mathrm{K}^{+} \rightarrow \pi^{+} v \bar{v}$ decay}

The measurement of the $\mathrm{K}^{+} \rightarrow \pi^{+} \nu v$ decay BR allows an extremely precise test of the Standard Model (SM). Given the absence of flavour changing neutral currents (FCNC) and its proportionality to $\mathrm{V}_{t s}^{*} \mathrm{~V}_{t d}$ CKM matrix element, the decay (Fig. 1) is highly suppressed within the SM. The theoretical BR prediction within the $\mathrm{SM}$ framework is extremely precise due to the possibility of relating the hadronic matrix element to the BR of the $\mathrm{K}^{+} \rightarrow \mathrm{e}^{+} \pi^{0} v$ decay. The latest prediction [4] yields:

$$
B R\left(K^{+} \rightarrow \pi^{+} \nu \bar{v}\right)=(7.81 \pm 0.75 \pm 0.29) \cdot 10^{-11}
$$

where the first uncertainty is related to input parameters (mainly $\mathrm{V}_{c b}$ ), while the second to long-distance QCD corrections.

Thanks to the small BR value and the very precise SM prediction, the $\mathrm{K}^{+} \rightarrow \pi^{+} \nu v$ channel can be used as a probe to detect new physics beyond the SM. The present $\mathrm{K}^{+} \rightarrow \pi^{+} v v$ BR experimental measurement is based on 7 candidate events collected by the E787 and E949 experiments [5]:

$$
B R\left(K^{+} \rightarrow \pi^{+} \nu \bar{\nu}\right)=\left(1.73_{-1.05}^{+1.15}\right) \cdot 10^{-10}
$$

\footnotetext{
${ }^{a}$ e-mail: stefano.venditti@cern.ch

${ }^{\mathrm{b}}$ On behalf of the NA62 collaboration.
}

This is an Open Access article distributed under the terms of the Creative Commons Attribution License 4.0, which permits unrestricted use, distribution, and reproduction in any medium, provided the original work is properly cited. 

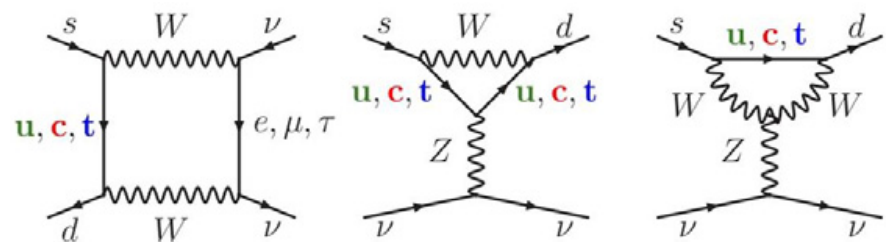

Figure 1. Feynman diagrams of the $\mathrm{K}^{+} \rightarrow \pi^{+} v v$ decay.

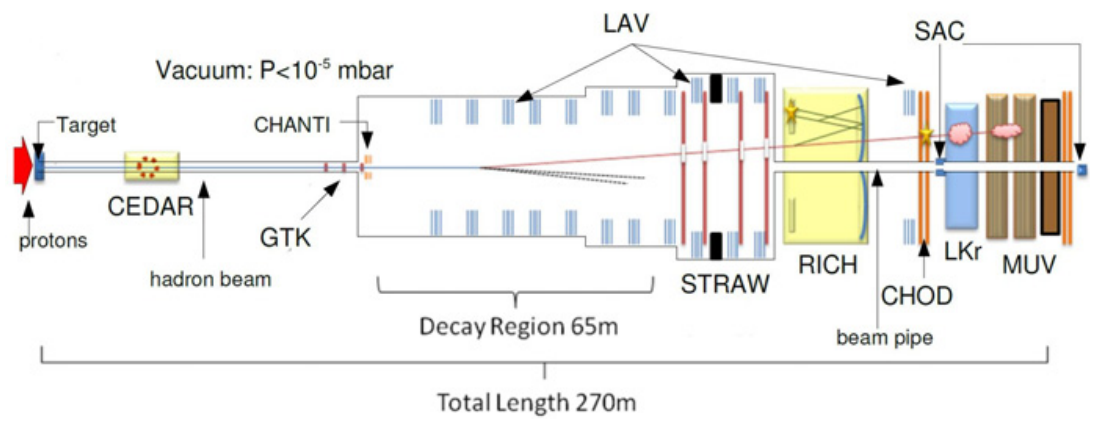

Figure 2. Layout of the NA62 experiment.

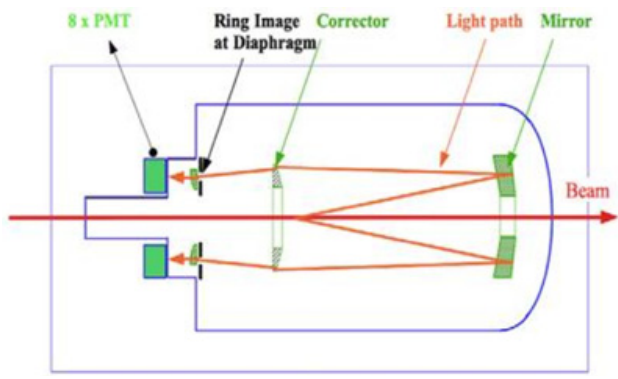

Figure 3. Ktag detector layout.

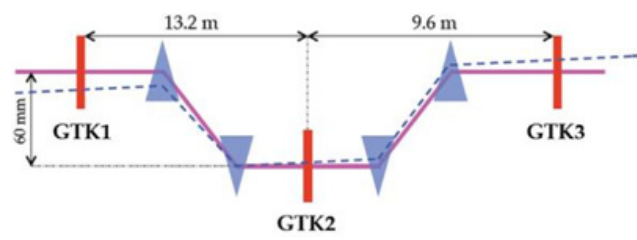

Figure 4. Gigatracker detector layout.

\section{The NA62 experiment}

The NA62 experiment at CERN will study several charged Kaon decays with unprecedented statistics. Its main goal is the measurement of the ultrarare $\mathrm{K}^{+} \rightarrow \pi^{+} \nu \bar{\nu}$ decay BR: the collection of $\mathrm{O}(100)$ events with $\sim 10 \%$ background and small systematics will allow to match the precision of the BR's experimental measurement to its theoretical estimation, to test the SM in a complementary way to LHC studies and to probe the presence of new physics.

A view of the NA62 experiment is shown in Fig. 2. SPS protons with an energy of $400 \mathrm{GeV} / \mathrm{c}$ inpinge on a Beryllium target; outgoing charged particles are selected in a $75 \pm 1 \% \mathrm{GeV} / \mathrm{c}$ momentum band. Before the decay region, Kaons are identified by the Ktag, a Helium-filled Cherenkov detector, with $10^{-3}$ inefficiency, and their momentum and direction measured with high precision by the Gigatracker, a silicon pixel detector made of 3 stations that will stand particle rates of $750 \mathrm{MHz}$. The layout of the two detectors is shown in Figs. 3 and 4 respectively. The CHANTI detector vetoes events triggered by the inelastic scattering of beam particles in the last Gigatracker station. 


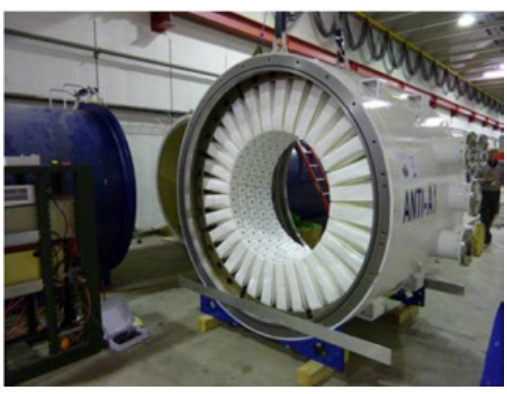

Figure 5. A LAV station.

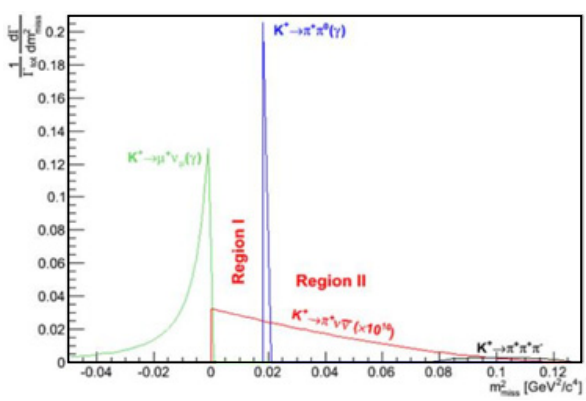

Figure 7. Reducible $\mathrm{K}^{+} \rightarrow \pi^{+} \nu \bar{v}$ background.

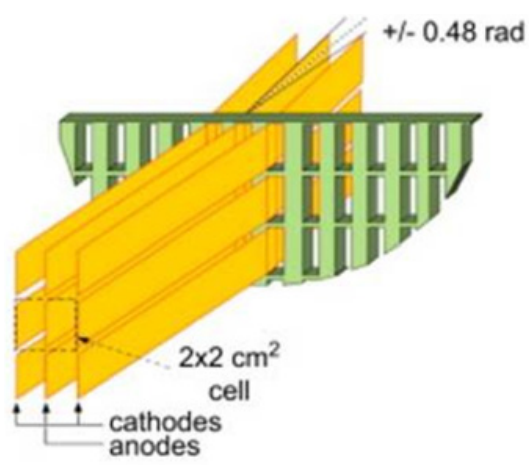

Figure 6. LKr cells and their typical zig-zag shape.

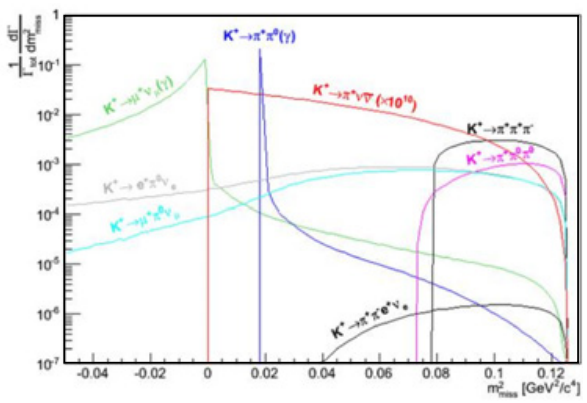

Figure 8. Irreducible $\mathrm{K}^{+} \rightarrow \pi^{+} v \bar{v}$ background.

The veto system aims at measuring particles (especially $\pi^{0} \mathrm{~s}$, whose detection inefficiency must be be $<10^{-8}$ ) from Kaon decays that would otherwise represent a BG for the $\mathrm{K}^{+} \rightarrow \pi^{+} \nu \bar{\nu}$ decay. It is made of:

- 12 Large Angle Veto stations (LAVs) along the beamline, each made of 4/5 rings of lead-glass crystals from the OPAL [6] calorimeter. The stations, one of which is shown in Fig. 5, were assembled at Laboratori Nazionali di Frascati (LNF) and then delivered to CERN;

- the Liquid Krypton (LKr) calorimeter, where electromagnetic showers of particles are measured with high precision. It is made of $10 \mathrm{~m}^{3}$ of liquid Krypton, divided into $132842 \times 2 \mathrm{~cm}^{2}$ cells by $\mathrm{Cu}$-Be ribbons. The cells, shown in Fig. 6, have a zig-zag shape and a projecting geometry towards the fiducial decay region. The signal, obtained by collecting the ionized electrons on the anode, is preamplified inside the LKr tank. Even though the LKr calorimeter was already existing, its readout system has been totally renewed [7] in order to cope with the rates requested by NA62;

- the IRC and SAC, two Shashlik-based detectors used to increase the LKr calorimeter acceptance at low angles and to detect photons from $\pi^{0} \mathrm{~s}$ decaying along the beamline, respectively.

The Straw system aims at measuring the momenta and directions of charged particles from Kaon decays with high precision. It is made of 4 chambers ( 2 before and 2 after a vertical magnetic field), each made of 4 views $(\mathrm{X}, \mathrm{Y}, \mathrm{U}, \mathrm{V})$ of 4 staggered straw layers each. The straws are $210 \mathrm{~cm}$ long, $9.8 \mathrm{~mm}$ diameter and filled with $\mathrm{Ar}+\mathrm{CO}_{2}$ gas (70\%:30\%). 
The RICH detector provides identification of charged Pions from Kaon decays at $5 \cdot 10^{-3}$ inefficiency. It is made of a tank filled with Helium at atmospheric pressure, where Cherenkov light emitted by particles is focused by hexagonal mirrors on two photomultiplier spots. The identification is given by the reconstruction of the Cherenkov ring.

The MUV system allows pion energy measurement (MUV1 +2 , iron/scintillator sandwich) and muon rejection (MUV3, scintillator tiles). The MUV3 will also be fundamental in the trigger chain to veto decays with muons, whose rate is $\sim 10 \mathrm{MHz}$.

The $\mathrm{BG}$ rejection of the $\mathrm{K}^{+} \rightarrow \pi^{+} \nu \bar{\nu}$ decay is performed by selecting two regions of the $\mathrm{M}_{\text {miss }}^{2}$ variable distribution, as shown in Fig. 7. The $\mathbf{M}_{\text {miss }}^{2}$ computation requires the high-precision measurement of the incoming Kaon and Pion four-momentum by the Gigatracker and by the Straw system, respectively:

$$
M_{m i s s}^{2}=\left(P_{K}-P_{\pi}\right)^{2} \simeq m_{K}^{2}\left(1-\frac{\left|P_{\pi}\right|}{\left|P_{K}\right|}\right)+m_{\pi}^{2}\left(1-\frac{\left|P_{K}\right|}{\left|P_{\pi}\right|}\right)-\left|P_{K}\right|\left|P_{\pi}\right| \theta_{K \pi}^{2} .
$$

Decays that cannot be reduced through the $\mathrm{M}_{m i s s}^{2}$ cut are shown in Fig. 8.

\section{The NA62 trigger system}

Three trigger levels will be implemented to reduce the $10 \mathrm{MHz}$ Kaon decays in the fiducial region down to $10 \mathrm{KHz}$ events, each level reducing the data load by about one order of magnitude.

The first trigger level (LOT) is FPGA-based and will use the TEL62 board [8], developed by the NA62 collaboration and based on the TELL1 board [9] used in the LHCb experiment. Each detector involved in the LOT decision will produce a trigger primitive; trigger primitives will be sent to a L0T supervisor, that will take the LOT decision. The LOT decision latency $(\sim 1 \mathrm{~ms})$ is fixed.

The other two trigger levels will be performed by a PC farm and their latency is not fixed. The second level trigger (L1T) algorithm will take a L1T decision using data collected after a LOT in $\sim 1 \mathrm{~s}$ and then send L1T signals to the detectors that didn't send their data to the PC farm at LOT level (e.g. the LKr). After this data is collected, the third trigger level algorithm (L2T) is applied on data, its latency being of the order of the SPS cycle. Events accepted by the L2T are written on disk.

\section{References}

[1] F. Hahn et al., NA62 Technical design document, CERN document NA62-10-07 (2010)

[2] C. Lazzeroni et al., Phys. Lett. B 719, 326-336 (2013)

[3] V. Fanti et al., Nucl. Instrum. Meth. A 574, 433-471 (2007)

[4] J. Brod, M. Gorbahn, E. Stamou, Phys. Rev. D 83, 034030 (2011)

[5] A.V. Artamonov et al., Phys. Rev. D 79, 092004 (2009)

[6] The OPAL experiment's website, http://opal.web.cern.ch/Opal/

[7] A. Ceccucci et al., JINST 9, C01047 (2014)

[8] B. Angelucci et al., IEEE Nucl. Sci. Symp. Conf. Rec. 823-826 (2011)

[9] The TELL1 project website, http://lphe.epfl.ch/tell1/ 